\title{
The tanh-coth method for some nonlinear pseudoparabolic equations with exact solutions
}

\section{Ömer Faruk Gözükızı ${ }^{*}$ and Şamil Akçağıl}

\section{"Correspondence:}

samilakcagil@hotmail.com

Department of Mathematics,

Sakarya University, Sakarya, Turkey

\begin{abstract}
We studied mostly important four nonlinear pseudoparabolic physical models: the Benjamin-Bona-Mahony-Peregrine-Burgers (BBMPB) equation, the

Oskolkov-Benjamin-Bona-Mahony-Burgers (OBBMB) equation, the one-dimensional Oskolkov equation and the generalised hyperelastic-rod wave equation. By using the tanh-coth method and symbolic computation system Maple, we have obtained abundant new solutions of these equations. The exact solutions show that the tanh-coth method is a powerful mathematical tool for solving nonlinear pseudoparabolic equations.
\end{abstract}

Keywords: nonlinear pseudoparabolic equation; Benjamin-Bona-Mahony-Peregrine-Burgers (BBMPB) equation; Oskolkov-Benjamin-Bona-Mahony-Burgers (OBBMB) equation; one-dimensional Oskolkov equation; generalised hyperelastic-rod wave equation; tanh-coth method

\section{Introduction}

Equations with a one-time derivative appearing in the highest order term are called pseudoparabolic and arise in many areas of mathematics and physics. They have been used, for instance, for fluid flow in fissured rock, consolidation of clay, shear in second-order fluids, thermodynamics and propagation of long waves of small amplitude. For more details, we refer the reader to [1-5] and references therein.

An important special case of pseudoparabolic-type equations is the generalised Benjamin-Bona-Mahony-Burgers (BBMB) equation

$$
u_{t}-u_{x x t}-\alpha u_{x x}+\gamma u_{x}+f(u)_{x}=0,
$$

where $u(x, t)$ represents the fluid velocity in the horizontal direction $x, \alpha$ is a positive constant, $\gamma$ is any given real constant and $f(u)$ is a $C^{2}$-smooth nonlinear function. For $f(u)_{x}=u u_{x}$ with $\alpha=0, \gamma=1$ in equation (1) was proposed as an alternative regularised long-wave equation by Peregrine [6] and Benjamin et al. [7] for the well-known Kortewegde Vries equation

$$
u_{t}+u_{x x t}+u_{x}+u u_{x}=0 .
$$

\section{Springer}

@ 2013 Gözükızl and Akçăgl|; licensee Springer. This is an Open Access article distributed under the terms of the Creative Commons Attribution License (http://creativecommons.org/licenses/by/2.0), which permits unrestricted use, distribution, and reproduction in any medium, provided the original work is properly cited. 
If we take $f(u)_{x}=\theta u u_{x}+\beta u_{x x x}$ in equation (1), then we obtain a general form of BenjaminBona-Mahony-Peregrine-Burgers (BBMPB) equation

$$
u_{t}-u_{x x t}-\alpha u_{x x}+\gamma u_{x}+\theta u u_{x}+\beta u_{x x x}=0 .
$$

Taking $\alpha=\beta=0$ in (3), we get the general form of the BBM equation as follows:

$$
u_{t}-u_{x x t}+\gamma u_{x}+\theta u u_{x}=0
$$

where $\gamma, \theta$ are constants and $\theta \neq 0$. Equation (3) includes several types of the BBM equation as seen in the literature. For more details, we refer the reader to [6-15]. We will study the general form of Benjamin-Bona-Mahony-Peregrine-Burgers (BBMPB) equation (3) using the tanh-coth method. We aim to extend the previous works especially in $[6,7]$ to make further progress for obtaining abundant new travelling wave solutions.

For $\beta=0$ in equation (3), we obtain a general form of the Oskolkov-Benjamin-BonaMahony-Burgers equation

$$
u_{t}-u_{x x t}-\alpha u_{x x}+\gamma u_{x}+\theta u u_{x}=0 .
$$

This nonlinear, one-dimensional and pseudoparabolic equation describes nonlinear surface waves that spread along the axis $O x$ and $\alpha u_{x x}$ is the viscosity term [16, 17]. In the literature the inverse scattering method has been thoroughly used to derive the multiple soliton solutions of equation (5) [8,18-22]. In this work we developed these solutions in a way that can be easily applied by using the tanh-coth method, which is less sophisticated than the inverse scattering method.

The equation

$$
u_{t}-\lambda u_{x x t}-\alpha u_{x x}+u u_{x}=0
$$

is a one-dimensional analogue of the Oskolkov system

$$
\left(1-\lambda \nabla^{2}\right) u_{t}=\alpha \nabla^{2} u-(u \cdot \nabla) u-\nabla^{2} p+f, \quad \nabla \cdot u=0 .
$$

This system describes the dynamics of an incompressible viscoelastic Kelvin-Voigt fluid. It was indicated in $[23,24]$ that the parameter $\lambda$ can be negative and the negativeness of the parameter $\lambda$ does not contradict the physical meaning of equation (7). We implemented the tanh-coth method to solve equation (6) and obtained new solutions which could not be attained in the past.

The generalised hyperelastic-rod wave equation

$$
u_{t}-u_{x x t}+\alpha u_{x}+2 \beta u u_{x}+3 \theta u^{2} u_{x}-\gamma u_{x} u_{x x}-u u_{x x x}=0
$$

was first introduced in [25], in which the global existence of dissipative solutions were established, where $\alpha, \beta, \theta$ and $\gamma$ are constant parameters. This equation includes many important physical models in mathematical physics. 
For $\beta=\frac{3}{2}, \theta=0, \gamma=2$, we obtain the Camassa-Holm $(\mathrm{CH})$ equation

$$
u_{t}-u_{x x t}+\alpha u_{x}+3 u u_{x}-2 u_{x} u_{x x}-u u_{x x x}=0,
$$

where $u$ is the fluid velocity in the direction $x$ (or, equivalently, the height of the water's free surface above a flat bottom), $\alpha$ is a constant related to the critical shallow water wave speed. Camassa-Holm equation has been studied in $[8,18]$ and explicit travelling-wave solutions were sought [19]. Besides, solitary wave solutions for modified forms of this equation were developed by Wazwaz [20].

Taking $\beta=2, \theta=0, \gamma=3$, equation (5) reduces to the Degasperis-Procesi (DP) equation

$$
u_{t}-u_{x x t}+\alpha u_{x}+4 u u_{x}-3 u_{x} u_{x x}-u u_{x x x}=0 .
$$

The recent study has revealed that the $\mathrm{CH}$ and DP equations can be used to describe the long-term dynamics of short surface waves [21, 22, 26].

For $\alpha=1, \beta=\frac{1}{2}, \theta=0, \gamma=3$, the equation (5) leads to the Fornberg-Whitham (FW) equation

$$
u_{t}-u_{x x t}+u_{x}+u u_{x}-3 u_{x} u_{x x}-u u_{x x x}=0 .
$$

The FW equation was used to study the qualitative behaviour of wave-breaking. A peaked solitary wave solution $u(x, t)=A e^{-\frac{1}{2}\left|x-\frac{4}{3} t\right|}$ of this type of equation was obtained by Fornberg and Whitham $[27,28]$. Using the tanh-coth method, we consider equation (8), which is a combined form of $\mathrm{CH}$, DP and FW equations, and obtain new exact solutions. These solutions can be seen as an improvement of the previously known data.

As stated before, pseudoparabolic-type equations arise in many areas of mathematics and physics to describe many physical phenomena. In recent years considerable attention has been paid to the study of pseudoparabolic-type equations, and to construct exact solutions for this type of equations, several methods, for instance, the tanh-coth method, have been developed. In [29, 30], we discussed some well-known Sobolev-type equations and pseudoparabolic equations and obtained new travelling wave solutions by using the tanh-coth method. Motivated by these studies, we employed the tanh-coth method to investigate new travelling wave solutions for the equations that were previously mentioned.

In what follows, we summarise the main features of the tanh-coth method as introduced in $[31,32]$, where more details and examples can be found.

\section{Outline of the tanh-coth method}

(i) First consider a general form of the nonlinear equation

$$
P\left(u, u_{t}, u_{x}, u_{x x}, \ldots\right)=0
$$

(ii) To find the travelling wave solution of equation (12), the wave variable $\xi=x-V t$ is introduced so that

$u(x, t)=U(\mu \xi)$ 
Based on this, one may use the following changes:

$$
\begin{aligned}
& \frac{\partial}{\partial t}=-V \frac{d}{d \xi}, \\
& \frac{\partial}{\partial x}=\mu \frac{d}{d \xi}, \\
& \frac{\partial^{2}}{\partial x^{2}}=\mu^{2} \frac{d^{2}}{d \xi^{2}}, \\
& \frac{\partial^{3}}{\partial x^{3}}=\mu^{3} \frac{d^{3}}{d \xi^{3}}
\end{aligned}
$$

and so on for other derivatives. Using (14) changes PDE (12) to an ODE

$$
Q\left(U, U^{\prime}, U^{\prime \prime}, \ldots\right)=0
$$

(iii) If all terms of the resulting ODE contain derivatives in $\xi$, then by integrating this equation, and by considering the constant of integration to be zero, one obtains a simplified ODE.

(iv) A new independent variable

$$
Y=\tanh (\mu \xi)
$$

is introduced that leads to the change of derivatives:

$$
\begin{aligned}
& \frac{d}{d \xi}=\mu\left(1-Y^{2}\right) \frac{d}{d Y}, \\
& \frac{d^{2}}{d \xi^{2}}=-2 \mu^{2} Y\left(1-Y^{2}\right) \frac{d}{d Y}+\mu^{2}\left(1-Y^{2}\right)^{2} \frac{d^{2}}{d Y^{2}}, \\
& \frac{d^{3}}{d \xi^{3}}=2 \mu^{3}\left(1-Y^{2}\right)\left(3 Y^{2}-1\right) \frac{d}{d Y}-6 \mu^{3} Y\left(1-Y^{2}\right)^{2} \frac{d^{2}}{d Y^{2}}+\mu^{3}\left(1-Y^{2}\right)^{3} \frac{d^{3}}{d Y^{3}},
\end{aligned}
$$

where other derivatives can be derived in a similar manner.

(v) The ansatz of the form

$$
U(\mu \xi)=S(Y)=\sum_{k=0}^{M} a_{k} Y^{k}+\sum_{k=1}^{M} b_{k} Y^{-k}
$$

is introduced where $M$ is a positive integer, in most cases, that will be determined. If $M$ is not an integer, then a transformation formula is used to overcome this difficulty. Substituting (17) and (18) into ODE (15) yields an equation in powers of $Y$.

(vi) To determine the parameter $M$, the linear terms of highest order in the resulting equation with the highest order nonlinear terms are balanced. With $M$ determined, one collects all the coefficients of powers of $Y$ in the resulting equation, where these coefficients have to vanish. This will give a system of algebraic equations involving $a_{k}$ and $b_{k}$ $(k=0, \ldots, M), V$, and $\mu$. Having determined these parameters, knowing that $M$ is a positive integer in most cases and using (18), one obtains an analytic solution in a closed form. 


\section{The Benjamin-Bona-Mahony-Peregrine-Burgers (BBMPB) equation}

The Benjamin-Bona-Mahony-Peregrine-Burgers (BBMPB) equation is given by

$$
u_{t}-u_{x x t}-\alpha u_{x x}+\gamma u_{x}+\theta u u_{x}+\beta u_{x x x}=0,
$$

where $\alpha$ is a positive constant, $\theta$ and $\beta$ are nonzero real numbers. Using the wave variable $\xi=x-V t$ in (19) then integrating this equation and considering the constant of integration to be zero, we obtain

$$
(-V+\gamma) U-\alpha U^{\prime}+\frac{\theta}{2} U^{2}+(V+\beta) U^{\prime \prime}=0
$$

Balancing $U^{2}$ with $U^{\prime \prime}$ in (20) gives $M=2$. The tanh-coth method admits the use of the finite expansion

$$
U(\mu \xi)=S(Y)=\sum_{k=0}^{2} a_{k} Y^{k}+\sum_{k=1}^{2} b_{k} Y^{-k}
$$

where $Y=\tanh (\mu \xi)$. Substituting (21) into (20) and collecting the coefficients of $Y$ and setting it equal to zero, we find the system of equation:

$$
\begin{aligned}
& Y^{8}: a_{2}^{2} \theta+12 V a_{2} \mu^{2}+12 a_{2} \beta \mu^{2}=0 \\
& Y^{7}: 2 a_{1} a_{2} \theta+4 a_{2} \alpha \mu+4 V a_{1} \mu^{2}+4 a_{1} \beta \mu^{2}=0 \\
& Y^{6}: 2 a_{2} \gamma+a_{1}^{2} \theta-2 V a_{2}+2 a_{0} a_{2} \theta+2 a_{1} \alpha \mu-16 V a_{2} \mu^{2}-16 a_{2} \beta \mu^{2}=0, \\
& Y^{5}: 2 a_{1} \gamma-2 V a_{1}+2 b_{1} a_{2} \theta+2 a_{0} a_{1} \theta-4 a_{2} \alpha \mu-4 V a_{1} \mu^{2}-4 a_{1} \beta \mu^{2}=0, \\
& Y^{4}: 2 a_{0} \gamma+a_{0}^{2} \theta-2 V a_{0}+2 b_{1} a_{1} \theta+2 b_{2} a_{2} \theta-2 b_{1} \alpha \mu-2 a_{1} \alpha \mu+4 V b_{2} \mu^{2}, \\
& \quad+4 V a_{2} \mu^{2}+4 b_{2} \beta \mu^{2}+4 a_{2} \beta \mu^{2}=0 \\
& Y^{3}: 2 b_{1} \gamma-2 V b_{1}+2 b_{1} a_{0} \theta+2 b_{2} a_{1} \theta-4 b_{2} \alpha \mu-4 V b_{1} \mu^{2}-4 b_{1} \beta \mu^{2}=0, \\
& Y^{2}: 2 b_{2} \gamma+b_{1}^{2} \theta-2 V b_{2}+2 b_{2} a_{0} \theta+2 b_{1} \alpha \mu-16 V b_{2} \mu^{2}-16 b_{2} \beta \mu^{2}=0, \\
& Y^{1}: 2 b_{1} b_{2} \theta+4 b_{2} \alpha \mu+4 V b_{1} \mu^{2}+4 b_{1} \beta \mu^{2}=0, \\
& Y^{0}: b_{2}^{2} \theta+12 V b_{2} \mu^{2}+12 b_{2} \beta \mu^{2}=0 .
\end{aligned}
$$

Using Maple gives eighteen sets of solutions:

$$
\begin{aligned}
& a_{0}=\frac{-\gamma-\beta}{\theta}, \quad a_{1}=\frac{\gamma+\beta}{\theta}, \quad a_{2}=b_{1}=b_{2}=0, \quad V=-\beta, \quad \mu=\frac{-\gamma-\beta}{2 \alpha}, \\
& a_{0}=a_{1}=\frac{-\gamma-\beta}{\theta}, \quad a_{2}=b_{1}=b_{2}=0, \quad V=-\beta, \quad \mu=\frac{\gamma+\beta}{2 \alpha}, \\
& a_{0}=\frac{-\gamma-\beta}{\theta}, \quad a_{1}=b_{1}=\frac{\gamma+\beta}{2 \theta}, \quad a_{2}=b_{2}=0, \quad V=-\beta, \quad \mu=\frac{-\gamma-\beta}{4 \alpha}, \\
& a_{0}=\frac{-\gamma-\beta}{\theta}, \quad a_{1}=a_{2}=b_{2}=0, \quad b_{1}=\frac{-\gamma-\beta}{\theta}, \quad V=-\beta, \quad \mu=\frac{\gamma+\beta}{2 \alpha}, \\
& a_{0}=\frac{-\gamma-\beta}{\theta}, \quad a_{1}=\frac{-\gamma-\beta}{2 \theta}, \quad a_{2}=b_{2}=0, \quad b_{1}=-\frac{\gamma+\beta}{2 \theta},
\end{aligned}
$$




$$
\begin{aligned}
& V=-\beta, \quad \mu=\frac{\gamma+\beta}{4 \alpha}, \\
& a_{0}=\frac{-\gamma-\beta}{\theta}, \quad a_{1}=a_{2}=b_{2}=0, \quad b_{1}=\frac{\gamma+\beta}{\theta}, \\
& V=-\beta, \quad \mu=-\frac{\gamma+\beta}{2 \alpha}, \\
& a_{0}=\frac{-10 \gamma \mu-10 \beta \mu+\alpha}{20 \mu \theta}, \quad a_{1}=-\frac{12 \alpha \mu}{5 \theta}, \quad a_{2}=-\frac{6 \alpha \mu}{5 \theta}, \quad b_{1}=b_{2}=0 \text {, } \\
& V=\frac{\frac{\alpha}{10}-\beta \mu}{\mu}, \quad \mu=\frac{5 \gamma+5 \beta \pm \sqrt{25 \gamma^{2}+50 \gamma \beta+25 \beta^{2}-24 \alpha^{2}}}{24 \alpha}, \\
& a_{0}=\frac{3(-10 \gamma \mu-10 \beta \mu+\alpha)}{20 \mu \theta}, \quad a_{1}=-\frac{12 \alpha \mu}{5 \theta}, \quad a_{2}=-\frac{6 \alpha \mu}{5 \theta}, \quad b_{1}=b_{2}=0 \text {, } \\
& V=\frac{\frac{\alpha}{10}-\beta \mu}{\mu}, \quad \mu=\frac{-5 \gamma-5 \beta \pm \sqrt{25 \gamma^{2}+50 \gamma \beta+25 \beta^{2}+24 \alpha^{2}}}{24 \alpha}, \\
& a_{0}=\frac{-10 \gamma \mu-10 \beta \mu-\alpha}{20 \mu \theta}, \quad a_{1}=-\frac{12 \alpha \mu}{5 \theta}, \quad a_{2}=\frac{6 \alpha \mu}{5 \theta}, \quad b_{1}=b_{2}=0 \text {, } \\
& V=\frac{-\frac{\alpha}{10}-\beta \mu}{\mu}, \quad \mu=\frac{-5 \gamma-5 \beta \pm \sqrt{25 \gamma^{2}+50 \gamma \beta+25 \beta^{2}-24 \alpha^{2}}}{24 \alpha}, \\
& a_{0}=\frac{3(-10 \gamma \mu-10 \beta \mu-\alpha)}{20 \mu \theta}, \quad a_{1}=-\frac{12 \alpha \mu}{5 \theta}, \quad a_{2}=\frac{6 \alpha \mu}{5 \theta}, \quad b_{1}=b_{2}=0 \text {, } \\
& V=\frac{-\frac{\alpha}{10}-\beta \mu}{\mu}, \quad \mu=\frac{5 \gamma+5 \beta \pm \sqrt{25 \gamma^{2}+50 \gamma \beta+25 \beta^{2}+24 \alpha^{2}}}{24 \alpha}, \\
& a_{0}=\frac{-10 \gamma \mu-10 \beta \mu+\alpha}{20 \mu \theta}, \quad a_{1}=a_{2}=0, \quad b_{1}=-\frac{12 \alpha \mu}{5 \theta}, \quad b_{2}=-\frac{6 \alpha \mu}{5 \theta} \text {, } \\
& V=\frac{\frac{\alpha}{10}-\beta \mu}{\mu}, \quad \mu=\frac{5 \gamma+5 \beta \pm \sqrt{25 \gamma^{2}+50 \gamma \beta+25 \beta^{2}-24 \alpha^{2}}}{24 \alpha}, \\
& a_{0}=\frac{3(-10 \gamma \mu-10 \beta \mu+\alpha)}{20 \mu \theta}, \quad a_{1}=a_{2}=0, \quad b_{1}=-\frac{12 \alpha \mu}{5 \theta}, \quad b_{2}=-\frac{6 \alpha \mu}{5 \theta} \text {, } \\
& V=\frac{\frac{\alpha}{10}-\beta \mu}{\mu}, \quad \mu=\frac{-5 \gamma-5 \beta \pm \sqrt{25 \gamma^{2}+50 \gamma \beta+25 \beta^{2}+24 \alpha^{2}}}{24 \alpha}, \\
& a_{0}=\frac{3(-10 \gamma \mu-10 \beta \mu-\alpha)}{20 \mu \theta}, \quad a_{1}=a_{2}=0, \quad b_{1}=-\frac{12 \alpha \mu}{5 \theta}, \quad b_{2}=\frac{6 \alpha \mu}{5 \theta}, \\
& V=\frac{-\frac{\alpha}{10}-\beta \mu}{\mu}, \quad \mu=\frac{5 \gamma+5 \beta \pm \sqrt{25 \gamma^{2}+50 \gamma \beta+25 \beta^{2}+24 \alpha^{2}}}{24 \alpha}, \\
& a_{0}=\frac{-10 \gamma \mu-10 \beta \mu-\alpha}{20 \mu \theta}, \quad a_{1}=a_{2}=0, \quad b_{1}=-\frac{12 \alpha \mu}{5 \theta}, \quad b_{2}=\frac{6 \alpha \mu}{5 \theta}, \\
& V=\frac{-\frac{\alpha}{10}-\beta \mu}{\mu}, \quad \mu=\frac{-5 \gamma-5 \beta \pm \sqrt{25 \gamma^{2}+50 \gamma \beta+25 \beta^{2}-24 \alpha^{2}}}{24 \alpha}, \\
& a_{0}=\frac{3(-20 \gamma \mu-20 \beta \mu-\alpha)}{80 \mu \theta}, \quad a_{1}=b_{1}=-\frac{12 \alpha \mu}{5 \theta}, \quad a_{2}=b_{2}=\frac{3 \alpha \mu}{5 \theta} \text {, } \\
& V=\frac{-\frac{\alpha}{20}-\beta \mu}{\mu}, \quad \mu=\frac{-5 \gamma-5 \beta \pm \sqrt{25 \gamma^{2}+50 \gamma \beta+25 \beta^{2}-24 \alpha^{2}}}{48 \alpha},
\end{aligned}
$$




$$
\begin{aligned}
& a_{0}=\frac{4(-5 \gamma \mu-5 \beta \mu-\alpha)}{16 \mu \theta}, \quad a_{1}=b_{1}=-\frac{12 \alpha \mu}{5 \theta}, \quad a_{2}=b_{2}=\frac{3 \alpha \mu}{5 \theta}, \\
& V=\frac{-\frac{\alpha}{20}-\beta \mu}{\mu}, \quad \mu=\frac{5 \gamma+5 \beta \pm \sqrt{25 \gamma^{2}+50 \gamma \beta+25 \beta^{2}+24 \alpha^{2}}}{48 \alpha}, \\
& a_{0}=\frac{3(-20 \gamma \mu-20 \beta \mu+\alpha)}{80 \mu \theta}, \quad a_{1}=b_{1}=-\frac{12 \alpha \mu}{5 \theta}, \quad a_{2}=b_{2}=-\frac{3 \alpha \mu}{5 \theta}, \\
& V=\frac{\frac{\alpha}{20}-\beta \mu}{\mu}, \quad \mu=\frac{5 \gamma+5 \beta \pm \sqrt{25 \gamma^{2}+50 \gamma \beta+25 \beta^{2}-24 \alpha^{2}}}{48 \alpha}, \\
& a_{0}=\frac{-20 \gamma \mu-20 \beta \mu+\alpha}{16 \mu \theta}, \quad a_{1}=b_{1}=-\frac{12 \alpha \mu}{5 \theta}, \quad a_{2}=b_{2}=-\frac{3 \alpha \mu}{5 \theta} \\
& V=\frac{\frac{\alpha}{20}-\beta \mu}{\mu}, \quad \mu=\frac{-5 \gamma-5 \beta \pm \sqrt{25 \gamma^{2}+50 \gamma \beta+25 \beta^{2}+24 \alpha^{2}}}{48 \alpha} .
\end{aligned}
$$

These sets give the solutions respectively:

$$
\begin{aligned}
& u_{1}(x, t)=\frac{-\gamma-\beta}{\theta}+\frac{\gamma+\beta}{\theta} \tanh \mu(x-V t), \\
& u_{2}(x, t)=\frac{-\gamma-\beta}{\theta}-\frac{\gamma+\beta}{\theta} \tanh \mu(x-V t), \\
& u_{3}(x, t)=\frac{-\gamma-\beta}{\theta}+\frac{\gamma+\beta}{2 \theta} \tanh \mu(x-V t)+\frac{\gamma+\beta}{2 \theta} \operatorname{coth} \mu(x-V t), \\
& u_{4}(x, t)=\frac{-\gamma-\beta}{\theta}-\frac{\gamma+\beta}{\theta} \operatorname{coth} \mu(x-V t), \\
& u_{5}(x, t)=\frac{-\gamma-\beta}{\theta}-\frac{\gamma+\beta}{\theta} \tanh \mu(x-V t)-\frac{\gamma+\beta}{2 \theta} \operatorname{coth} \mu(x-V t), \\
& u_{6}(x, t)=\frac{-\gamma-\beta}{\theta}+\frac{\gamma+\beta}{\theta} \operatorname{coth} \mu(x-V t), \\
& u_{7}(x, t)=\frac{-10 \gamma \mu-10 \beta \mu+\alpha}{20 \mu \theta}-\frac{12 \alpha \mu}{5 \theta} \tanh \mu(x-V t)-\frac{6 \alpha \mu}{5 \theta} \tanh ^{2} \mu(x-V t), \\
& u_{8}(x, t)=\frac{3(-10 \gamma \mu-10 \beta \mu+\alpha)}{20 \mu \theta}-\frac{12 \alpha \mu}{5 \theta} \tanh \mu(x-V t)-\frac{6 \alpha \mu}{5 \theta} \tanh ^{2} \mu(x-V t) \text {, } \\
& u_{9}(x, t)=\frac{-10 \gamma \mu-10 \beta \mu-\alpha}{20 \mu \theta}-\frac{12 \alpha \mu}{5 \theta} \tanh \mu(x-V t)+\frac{6 \alpha \mu}{5 \theta} \tanh ^{2} \mu(x-V t) \\
& -\frac{12 \alpha \mu}{5} \operatorname{coth} \mu(x-V t)-\frac{3 \alpha \mu}{5} \operatorname{coth}^{2} \mu(x-V t), \\
& u_{10}(x, t)=\frac{3(-10 \gamma \mu-10 \beta \mu-\alpha)}{20 \mu \theta}-\frac{12 \alpha \mu}{5 \theta} \tanh \mu(x-V t)+\frac{6 \alpha \mu}{5 \theta} \tanh ^{2} \mu(x-V t) \text {, } \\
& u_{11}(x, t)=\frac{-10 \gamma \mu-10 \beta \mu+\alpha}{20 \mu \theta}-\frac{12 \alpha \mu}{5 \theta} \operatorname{coth} \mu(x-V t)-\frac{6 \alpha \mu}{5 \theta} \operatorname{coth}^{2} \mu(x-V t) \text {, } \\
& u_{12}(x, t)=\frac{3(-10 \gamma \mu-10 \beta \mu+\alpha)}{20 \mu \theta}-\frac{12 \alpha \mu}{5 \theta} \operatorname{coth} \mu(x-V t)-\frac{6 \alpha \mu}{5 \theta} \operatorname{coth}^{2} \mu(x-V t) \text {, } \\
& u_{13}(x, t)=\frac{3(-10 \gamma \mu-10 \beta \mu-\alpha)}{20 \mu \theta}-\frac{12 \alpha \mu}{5 \theta} \operatorname{coth} \mu(x-V t)+\frac{6 \alpha \mu}{5 \theta} \operatorname{coth}^{2} \mu(x-V t) \text {, } \\
& u_{14}(x, t)=\frac{-10 \gamma \mu-10 \beta \mu-\alpha}{20 \mu \theta}-\frac{12 \alpha \mu}{5 \theta} \operatorname{coth} \mu(x-V t)+\frac{6 \alpha \mu}{5 \theta} \operatorname{coth}^{2} \mu(x-V t) \text {, }
\end{aligned}
$$




$$
\begin{aligned}
u_{15}(x, t)= & \frac{3(-20 \gamma \mu-20 \beta \mu-\alpha)}{80 \mu \theta}-\frac{12 \alpha \mu}{5 \theta} \tanh \mu(x-V t)+\frac{3 \alpha \mu}{5 \theta} \tanh ^{2} \mu(x-V t) \\
& -\frac{12 \alpha \mu}{5 \theta} \operatorname{coth} \mu(x-V t)+\frac{3 \alpha \mu}{5 \theta} \operatorname{coth}^{2} \mu(x-V t), \\
u_{16}(x, t)= & \frac{4(-5 \gamma \mu-5 \beta \mu-\alpha)}{16 \mu \theta}-\frac{12 \alpha \mu}{5 \theta} \tanh \mu(x-V t)+\frac{3 \alpha \mu}{5 \theta} \tanh ^{2} \mu(x-V t) \\
& -\frac{12 \alpha \mu}{5 \theta} \operatorname{coth} \mu(x-V t)+\frac{3 \alpha \mu}{5 \theta} \operatorname{coth}^{2} \mu(x-V t), \\
u_{17}(x, t)= & \frac{3(-20 \gamma \mu-20 \beta \mu+\alpha)}{80 \mu \theta}-\frac{12 \alpha \mu}{5 \theta} \tanh \mu(x-V t)-\frac{3 \alpha \mu}{5 \theta} \tanh ^{2} \mu(x-V t) \\
& -\frac{12 \alpha \mu}{5 \theta} \operatorname{coth} \mu(x-V t)-\frac{3 \alpha \mu}{5 \theta} \operatorname{coth}^{2} \mu(x-V t), \\
& -20 \gamma \mu-20 \beta \mu+\alpha \\
16 \mu \theta & \frac{12 \alpha \mu}{5 \theta} \tanh ^{2} \mu(x-V t)-\frac{3 \alpha \mu}{5 \theta} \tanh ^{2} \mu(x-V t) \\
& -\frac{12 \alpha \mu}{5 \theta} \operatorname{coth} \mu(x-V t)-\frac{3 \alpha \mu}{5 \theta} \operatorname{coth}^{2} \mu(x-V t) .
\end{aligned}
$$

\section{The Oskolkov-Benjamin-Bona-Mahony-Burgers (OBBMB) equation}

We consider the Oskolkov-Benjamin-Bona-Mahony-Burgers (OBBMB) equation

$$
u_{t}-u_{x x t}-\alpha u_{x x}+\gamma u_{x}+\theta u u_{x}=0
$$

where $\alpha$ is positive and $\theta$ is a nonzero constant. Using the wave variable $\xi=x-V t$ in (25) then integrating this equation and considering the constant of integration to be zero, we obtain

$$
(-V+\gamma) U+\frac{\theta}{2} U^{2}-\alpha U^{\prime}+V U^{\prime \prime}=0
$$

Balancing the second term with the last term in (26) gives $M=2$. Using the finite expansion

$$
U(\mu \xi)=S(Y)=\sum_{k=0}^{2} a_{k} Y^{k}+\sum_{k=1}^{2} b_{k} Y^{-k}
$$

where $Y=\tanh (\mu \xi)$. Substituting (27) into (26) and collecting the coefficients of $Y$ and setting it equal to zero, we find the system of equations:

$$
\begin{aligned}
& Y^{8}: a_{2}^{2} \theta+12 V a_{2} \mu^{2}=0, \\
& Y^{7}: 2 a_{1} a_{2} \theta+4 a_{2} \alpha \mu+4 V a_{1} \mu^{2}=0, \\
& Y^{6}: 2 a_{2} \gamma+a_{1}^{2} \theta-2 V a_{2}+2 a_{0} a_{2} \theta+2 a_{1} \alpha \mu-16 V a_{2} \mu^{2}=0, \\
& Y^{5}: 2 a_{1} \gamma-2 V a_{1}+2 b_{1} a_{2} \theta+2 a_{0} a_{1} \theta-4 a_{2} \alpha \mu-4 V a_{1} \mu^{2}=0, \\
& Y^{4}: 2 a_{0} \gamma+a_{0}^{2} \theta-2 V a_{0}+2 b_{1} a_{1} \theta+2 b_{2} a_{2} \theta-2 b_{1} \alpha \mu-2 a_{1} \alpha \mu \\
& \quad+4 V b_{2} \mu^{2}+4 V a_{2} \mu^{2}=0, \\
& Y^{3}: 2 b_{1} \gamma-2 V b_{1}+2 b_{1} a_{0} \theta+2 b_{2} a_{1} \theta-4 b_{2} \alpha \mu-4 V b_{1} \mu^{2}=0,
\end{aligned}
$$




$$
\begin{aligned}
& Y^{2}: 2 b_{2} \gamma+b_{1}^{2} \theta-2 V b_{2}+2 b_{2} a_{0} \theta+2 b_{1} \alpha \mu-16 V b_{2} \mu^{2}=0, \\
& Y^{1}: 2 b_{1} b_{2} \theta+4 b_{2} \alpha \mu+4 V b_{1} \mu^{2}=0, \\
& Y^{0}: b_{2}^{2} \theta+12 V b_{2} \mu^{2}=0 .
\end{aligned}
$$

Maple gives twelve sets of solutions:

$$
\begin{aligned}
& a_{0}=\frac{-10 \gamma \mu+\alpha}{20 \mu \theta}, \quad a_{1}=-\frac{12 \alpha \mu}{5 \theta}, \quad a_{2}=-\frac{6 \alpha \mu}{5 \theta}, \quad b_{1}=b_{2}=0 \text {, } \\
& V=\frac{\alpha}{10 \mu}, \quad \mu=\frac{5 \gamma \pm \sqrt{25 \gamma^{2}-24 \alpha^{2}}}{24 \alpha}, \\
& a_{0}=\frac{3(-10 \gamma \mu+\alpha)}{20 \mu \theta}, \quad a_{1}=-\frac{12 \alpha \mu}{5 \theta}, \quad a_{2}=-\frac{6 \alpha \mu}{5 \theta}, \quad b_{1}=b_{2}=0, \\
& V=\frac{\alpha}{10 \mu}, \quad \mu=\frac{-5 \gamma \pm \sqrt{25 \gamma^{2}+24 \alpha^{2}}}{24 \alpha}, \\
& a_{0}=\frac{-10 \gamma \mu-\alpha}{20 \mu \theta}, \quad a_{1}=-\frac{12 \alpha \mu}{5 \theta}, \quad a_{2}=\frac{6 \alpha \mu}{5 \theta}, \quad b_{1}=b_{2}=0, \\
& V=-\frac{\alpha}{10 \mu}, \quad \mu=\frac{-5 \gamma \pm \sqrt{25 \gamma^{2}-24 \alpha^{2}}}{24 \alpha}, \\
& a_{0}=\frac{3(-10 \gamma \mu-\alpha)}{20 \mu \theta}, \quad a_{1}=-\frac{12 \alpha \mu}{5 \theta}, \quad a_{2}=\frac{6 \alpha \mu}{5 \theta}, \quad b_{1}=b_{2}=0 \text {, } \\
& V=-\frac{\alpha}{10 \mu}, \quad \mu=\frac{5 \gamma \pm \sqrt{25 \gamma^{2}+24 \alpha^{2}}}{24 \alpha}, \\
& a_{0}=\frac{-10 \gamma \mu+\alpha}{20 \mu \theta}, \quad a_{1}=a_{2}=0, \quad b_{1}=-\frac{12 \alpha \mu}{5 \theta}, \quad b_{2}=-\frac{6 \alpha \mu}{5 \theta} \text {, } \\
& V=\frac{\alpha}{10 \mu}, \quad \mu=\frac{5 \gamma \pm \sqrt{25 \gamma^{2}-24 \alpha^{2}}}{24 \alpha}, \\
& a_{0}=\frac{3(-10 \gamma \mu+\alpha)}{20 \mu \theta}, \quad a_{1}=a_{2}=0, \quad b_{1}=-\frac{12 \alpha \mu}{5 \theta}, \quad b_{2}=-\frac{6 \alpha \mu}{5 \theta}, \\
& V=\frac{\alpha}{10 \mu}, \quad \mu=\frac{-5 \gamma \pm \sqrt{25 \gamma^{2}+24 \alpha^{2}}}{24 \alpha}, \\
& a_{0}=\frac{3(-10 \gamma \mu-\alpha)}{20 \mu \theta}, \quad a_{1}=a_{2}=0, \quad b_{1}=-\frac{12 \alpha \mu}{5 \theta}, \quad b_{2}=\frac{6 \alpha \mu}{5 \theta} \text {, } \\
& V=-\frac{\alpha}{10 \mu}, \quad \mu=\frac{5 \gamma \pm \sqrt{25 \gamma^{2}+24 \alpha^{2}}}{24 \alpha}, \\
& a_{0}=\frac{-10 \gamma \mu-\alpha}{20 \mu \theta}, \quad a_{1}=a_{2}=0, \quad b_{1}=-\frac{12 \alpha \mu}{5 \theta}, \quad b_{2}=\frac{6 \alpha \mu}{5 \theta}, \\
& V=-\frac{\alpha}{10 \mu}, \quad \mu=\frac{-5 \gamma \pm \sqrt{25 \gamma^{2}-24 \alpha^{2}}}{24 \alpha}, \\
& a_{0}=\frac{3(-20 \gamma \mu-\alpha)}{80 \mu \theta}, \quad a_{1}=b_{1}=-\frac{12 \alpha \mu}{5 \theta}, \quad a_{2}=b_{2}=\frac{3 \alpha \mu}{5 \theta}, \\
& V=-\frac{\alpha}{20 \mu}, \quad \mu=\frac{-5 \gamma \pm \sqrt{25 \gamma^{2}-24 \alpha^{2}}}{48 \alpha},
\end{aligned}
$$




$$
\begin{array}{ll}
a_{0}=\frac{4(-5 \gamma \mu-\alpha)}{16 \mu \theta}, \quad a_{1}=b_{1}=-\frac{12 \alpha \mu}{5 \theta}, \quad a_{2}=b_{2}=\frac{3 \alpha \mu}{5 \theta}, \\
V=-\frac{\alpha}{20 \mu}, \quad \mu=\frac{5 \gamma \pm \sqrt{25 \gamma^{2}+24 \alpha^{2}}}{48 \alpha}, \\
a_{0}=\frac{3(-20 \gamma \mu+\alpha)}{80 \mu \theta}, \quad a_{1}=b_{1}=-\frac{12 \alpha \mu}{5 \theta}, \quad a_{2}=b_{2}=-\frac{3 \alpha \mu}{5 \theta}, \\
V=\frac{\alpha}{20 \mu}, \quad \mu=\frac{5 \gamma \pm \sqrt{25 \gamma^{2}-24 \alpha^{2}}}{48 \alpha}, \\
a_{0}=\frac{-20 \gamma \mu+\alpha}{16 \mu \theta}, \quad a_{1}=b_{1}=-\frac{12 \alpha \mu}{5 \theta}, & a_{2}=b_{2}=-\frac{3 \alpha \mu}{5 \theta}, \\
V=\frac{\alpha}{20 \mu}, \quad \mu=\frac{-5 \gamma \pm \sqrt{25 \gamma^{2}+24 \alpha^{2}}}{48 \alpha} . &
\end{array}
$$

These give the following solutions:

$$
\begin{aligned}
& u_{1}(x, t)=\frac{-10 \gamma \mu-10 \beta \mu+\alpha}{20 \mu \theta}-\frac{12 \alpha \mu}{5 \theta} \tanh \mu(x-V t)-\frac{6 \alpha \mu}{5 \theta} \tanh ^{2} \mu(x-V t) \text {, } \\
& u_{2}(x, t)=\frac{3(-10 \gamma \mu-10 \beta \mu+\alpha)}{20 \mu \theta}-\frac{12 \alpha \mu}{5 \theta} \tanh \mu(x-V t)-\frac{6 \alpha \mu}{5 \theta} \tanh ^{2} \mu(x-V t), \\
& u_{3}(x, t)=\frac{-10 \gamma \mu-10 \beta \mu-\alpha}{20 \mu \theta}-\frac{12 \alpha \mu}{5 \theta} \tanh \mu(x-V t)+\frac{6 \alpha \mu}{5 \theta} \tanh ^{2} \mu(x-V t) \\
& -\frac{12 \alpha \mu}{5} \operatorname{coth} \mu(x-V t)-\frac{3 \alpha \mu}{5} \operatorname{coth}^{2} \mu(x-V t), \\
& u_{4}(x, t)=\frac{3(-10 \gamma \mu-10 \beta \mu-\alpha)}{20 \mu \theta}-\frac{12 \alpha \mu}{5 \theta} \tanh \mu(x-V t)+\frac{6 \alpha \mu}{5 \theta} \tanh ^{2} \mu(x-V t), \\
& u_{5}(x, t)=\frac{-10 \gamma \mu-10 \beta \mu+\alpha}{20 \mu \theta}-\frac{12 \alpha \mu}{5 \theta} \operatorname{coth} \mu(x-V t)-\frac{6 \alpha \mu}{5 \theta} \operatorname{coth}^{2} \mu(x-V t), \\
& u_{6}(x, t)=\frac{3(-10 \gamma \mu-10 \beta \mu+\alpha)}{20 \mu \theta}-\frac{12 \alpha \mu}{5 \theta} \operatorname{coth} \mu(x-V t)-\frac{6 \alpha \mu}{5 \theta} \operatorname{coth}^{2} \mu(x-V t) \text {, } \\
& u_{7}(x, t)=\frac{3(-10 \gamma \mu-10 \beta \mu-\alpha)}{20 \mu \theta}-\frac{12 \alpha \mu}{5 \theta} \operatorname{coth} \mu(x-V t)+\frac{6 \alpha \mu}{5 \theta} \operatorname{coth}^{2} \mu(x-V t), \\
& u_{8}(x, t)=\frac{-10 \gamma \mu-10 \beta \mu-\alpha}{20 \mu \theta}-\frac{12 \alpha \mu}{5 \theta} \operatorname{coth} \mu(x-V t)+\frac{6 \alpha \mu}{5 \theta} \operatorname{coth}^{2} \mu(x-V t), \\
& u_{9}(x, t)=\frac{3(-20 \gamma \mu-20 \beta \mu-\alpha)}{80 \mu \theta}-\frac{12 \alpha \mu}{5 \theta} \tanh \mu(x-V t)+\frac{3 \alpha \mu}{5 \theta} \tanh ^{2} \mu(x-V t) \\
& -\frac{12 \alpha \mu}{5 \theta} \operatorname{coth} \mu(x-V t)+\frac{3 \alpha \mu}{5 \theta} \operatorname{coth}^{2} \mu(x-V t), \\
& u_{10}(x, t)=\frac{4(-5 \gamma \mu-5 \beta \mu-\alpha)}{16 \mu \theta}-\frac{12 \alpha \mu}{5 \theta} \tanh \mu(x-V t)+\frac{3 \alpha \mu}{5 \theta} \tanh ^{2} \mu(x-V t) \\
& -\frac{12 \alpha \mu}{5 \theta} \operatorname{coth} \mu(x-V t)+\frac{3 \alpha \mu}{5 \theta} \operatorname{coth}^{2} \mu(x-V t), \\
& u_{11}(x, t)=\frac{3(-20 \gamma \mu-20 \beta \mu+\alpha)}{80 \mu \theta}-\frac{12 \alpha \mu}{5 \theta} \tanh \mu(x-V t)-\frac{3 \alpha \mu}{5 \theta} \tanh ^{2} \mu(x-V t) \\
& -\frac{12 \alpha \mu}{5 \theta} \operatorname{coth} \mu(x-V t)-\frac{3 \alpha \mu}{5 \theta} \operatorname{coth}^{2} \mu(x-V t),
\end{aligned}
$$




$$
\begin{aligned}
u_{12}(x, t)= & \frac{-20 \gamma \mu-20 \beta \mu+\alpha}{16 \mu \theta}-\frac{12 \alpha \mu}{5 \theta} \tanh \mu(x-V t)-\frac{3 \alpha \mu}{5 \theta} \tanh ^{2} \mu(x-V t) \\
& -\frac{12 \alpha \mu}{5 \theta} \operatorname{coth} \mu(x-V t)-\frac{3 \alpha \mu}{5 \theta} \operatorname{coth}^{2} \mu(x-V t) .
\end{aligned}
$$

As can be seen easily, these solutions can be obtained by taking $\beta=0$ in the solutions of previous equations.

\section{The one-dimensional Oskolkov equation}

The one-dimensional Oskolkov equation is given by

$$
u_{t}-\lambda u_{x x t}-\alpha u_{x x}+u u_{x}=0
$$

We will investigate the equation for $\lambda \neq 0$ and $\alpha \in \mathbb{R}$. Using the wave variable $\xi=x-V t$ in (31) then integrating this equation and considering the constant of integration to be zero, we obtain

$$
-V U+\lambda U^{\prime \prime}-\alpha U^{\prime}+\frac{1}{2} U^{2}=0
$$

Balancing $U^{2}$ with $U^{\prime \prime}$ in (32) gives $M=2$. The tanh-coth method admits the use of the finite expansion

$$
U(\mu \xi)=S(Y)=\sum_{k=0}^{2} a_{k} Y^{k}+\sum_{k=1}^{2} b_{k} Y^{-k}
$$

where $Y=\tanh (\mu \xi)$. Substituting (33) into (32) and collecting the coefficients of $Y$ and setting it equal to zero, we find the system of equations:

$$
\begin{aligned}
& Y^{8}: a_{2}^{2}+12 \lambda a_{2} \mu^{2}=0, \\
& Y^{7}: 4 a_{1} \lambda \mu^{2}+4 a_{2} \alpha \mu+2 a_{1} a_{2}=0, \\
& Y^{6}: a_{1}^{2}+2 \alpha a_{1} \mu-16 a_{2} \lambda \mu^{2}-2 V a_{2}+2 a_{0} a_{2}=0, \\
& Y^{5}: 2 b_{1} a_{2}-4 a_{2} \alpha \mu-2 V a_{1}-4 a_{1} \lambda \mu^{2}+2 a_{0} a_{1}=0, \\
& Y^{4}: 2 b_{1} a_{1}-2 V a_{0}+2 b_{2} a_{2}+a_{0}^{2}-2 b_{1} \alpha \mu-2 a_{1} \alpha \mu+4 b_{2} \lambda \mu^{2}+4 a_{2} \lambda \mu^{2}=0, \\
& Y^{3}: 2 b_{1} a_{0}-4 b_{2} \alpha \mu-2 V b_{1}-4 b_{1} \lambda \mu^{2}+2 b_{2} a_{1}=0, \\
& Y^{2}: b_{1}^{2}+2 \alpha b_{1} \mu-16 b_{2} \lambda \mu^{2}-2 V b_{2}+2 b_{2} a_{0}=0, \\
& Y^{1}: 4 b_{1} \lambda \mu^{2}+4 b_{2} \alpha \mu+2 b_{1} b_{2}=0, \\
& Y^{0}: b_{2}^{2}+12 \lambda b_{2} \mu^{2}=0 .
\end{aligned}
$$

Solving this system, we find the following sets of solutions:

$$
\begin{array}{ll}
a_{0}=\frac{9 \alpha^{2}}{25 \lambda}, & a_{1}=-\frac{6 \alpha^{2}}{25 \lambda}, \quad a_{2}=-\frac{3 \alpha^{2}}{25 \lambda}, \quad b_{1}=b_{2}=0, \\
V=\frac{6 \alpha^{2}}{25 \lambda}, & \mu=\frac{\alpha}{10 \lambda},
\end{array}
$$




$$
\begin{aligned}
& a_{0}=-\frac{3 \alpha^{2}}{25 \lambda}, \quad a_{1}=-\frac{6 \alpha^{2}}{25 \lambda}, \quad a_{2}=-\frac{3 \alpha^{2}}{25 \lambda}, \quad b_{1}=b_{2}=0 \\
& V=-\frac{6 \alpha^{2}}{25 \lambda}, \quad \mu=\frac{\alpha}{10 \lambda} \\
& a_{0}=\frac{9 \alpha^{2}}{25 \lambda}, \quad a_{1}=\frac{6 \alpha^{2}}{25 \lambda}, \quad a_{2}=-\frac{3 \alpha^{2}}{25 \lambda}, \quad b_{1}=b_{2}=0, \\
& V=\frac{6 \alpha^{2}}{25 \lambda}, \quad \mu=-\frac{\alpha}{10 \lambda} \text {, } \\
& a_{0}=-\frac{3 \alpha^{2}}{25 \lambda}, \quad a_{1}=\frac{6 \alpha^{2}}{25 \lambda}, \quad a_{2}=-\frac{3 \alpha^{2}}{25 \lambda}, \quad b_{1}=b_{2}=0 \\
& V=-\frac{6 \alpha^{2}}{25 \lambda}, \quad \mu=-\frac{\alpha}{10 \lambda} \\
& a_{0}=\frac{9 \alpha^{2}}{25 \lambda}, \quad a_{1}=a_{2}=0, \quad b_{1}=\frac{6 \alpha^{2}}{25 \lambda}, \quad b_{2}=-\frac{3 \alpha^{2}}{25 \lambda} \text {, } \\
& V=\frac{6 \alpha^{2}}{25 \lambda}, \quad \mu=-\frac{\alpha}{10 \lambda}, \\
& a_{0}=-\frac{3 \alpha^{2}}{25 \lambda}, \quad a_{1}=a_{2}=0, \quad b_{1}=\frac{6 \alpha^{2}}{25 \lambda}, \quad b_{2}=-\frac{3 \alpha^{2}}{25 \lambda}, \\
& V=-\frac{6 \alpha^{2}}{25 \lambda}, \quad \mu=-\frac{\alpha}{10 \lambda} \\
& a_{0}=\frac{3 \alpha^{2}}{10 \lambda}, \quad a_{1}=-\frac{3 \alpha^{2}}{25 \lambda}, \quad a_{2}=-\frac{3 \alpha^{2}}{100 \lambda}, \quad b_{1}=-\frac{3 \alpha^{2}}{25 \lambda}, \quad b_{2}=-\frac{3 \alpha^{2}}{100 \lambda}, \\
& V=\frac{6 \alpha^{2}}{25 \lambda}, \quad \mu=\frac{\alpha}{20 \lambda} \\
& a_{0}=-\frac{9 \alpha^{2}}{50 \lambda}, \quad a_{1}=-\frac{3 \alpha^{2}}{25 \lambda}, \quad a_{2}=-\frac{3 \alpha^{2}}{100 \lambda}, \quad b_{1}=-\frac{3 \alpha^{2}}{25 \lambda}, \quad b_{2}=-\frac{3 \alpha^{2}}{100 \lambda}, \\
& V=-\frac{6 \alpha^{2}}{25 \lambda}, \quad \mu=\frac{\alpha}{20 \lambda}, \\
& a_{0}=\frac{3 \alpha^{2}}{10 \lambda}, \quad a_{1}=\frac{3 \alpha^{2}}{25 \lambda}, \quad a_{2}=-\frac{3 \alpha^{2}}{100 \lambda}, \quad b_{1}=\frac{3 \alpha^{2}}{25 \lambda}, \quad b_{2}=-\frac{3 \alpha^{2}}{100 \lambda}, \\
& V=\frac{6 \alpha^{2}}{25 \lambda}, \quad \mu=-\frac{\alpha}{20 \lambda} \\
& a_{0}=-\frac{9 \alpha^{2}}{50 \lambda}, \quad a_{1}=\frac{3 \alpha^{2}}{25 \lambda}, \quad a_{2}=-\frac{3 \alpha^{2}}{100 \lambda}, \quad b_{1}=\frac{3 \alpha^{2}}{25 \lambda}, \quad b_{2}=-\frac{3 \alpha^{2}}{100 \lambda}, \\
& V=-\frac{6 \alpha^{2}}{25 \lambda}, \quad \mu=-\frac{\alpha}{20 \lambda} \\
& a_{0}=\frac{9 \alpha^{2}}{25 \lambda}, \quad a_{1}=a_{2}=0, \quad b_{1}=-\frac{6 \alpha^{2}}{25 \lambda}, \quad b_{2}=-\frac{3 \alpha^{2}}{25 \lambda}, \\
& V=\frac{6 \alpha^{2}}{25 \lambda}, \quad \mu=\frac{\alpha}{10 \lambda}, \\
& a_{0}=-\frac{3 \alpha^{2}}{25 \lambda}, \quad a_{1}=a_{2}=0, \quad b_{1}=-\frac{6 \alpha^{2}}{25 \lambda}, \quad b_{2}=-\frac{3 \alpha^{2}}{25 \lambda}, \\
& V=-\frac{6 \alpha^{2}}{25 \lambda}, \quad \mu=\frac{\alpha}{10 \lambda}
\end{aligned}
$$


These sets give the solutions respectively:

$$
\begin{aligned}
& u_{1}(x, t)=\frac{9 \alpha^{2}}{25 \lambda}-\frac{6 \alpha^{2}}{25 \lambda} \tanh \mu(x-V t)-\frac{3 \alpha^{2}}{25 \lambda} \tanh ^{2} \mu(x-V t) \text {, } \\
& u_{2}(x, t)=-\frac{3 \alpha^{2}}{25 \lambda}-\frac{6 \alpha^{2}}{25 \lambda} \tanh \mu(x-V t)-\frac{3 \alpha^{2}}{25 \lambda} \tanh ^{2} \mu(x-V t) \text {, } \\
& u_{3}(x, t)=\frac{9 \alpha^{2}}{25 \lambda}+\frac{6 \alpha^{2}}{25 \lambda} \tanh \mu(x-V t)-\frac{3 \alpha^{2}}{25 \lambda} \tanh ^{2} \mu(x-V t), \\
& u_{4}(x, t)=-\frac{3 \alpha^{2}}{25 \lambda}+\frac{6 \alpha^{2}}{25 \lambda} \tanh \mu(x-V t)-\frac{3 \alpha^{2}}{25 \lambda} \tanh ^{2} \mu(x-V t) \text {, } \\
& u_{5}(x, t)=\frac{9 \alpha^{2}}{25 \lambda}+\frac{6 \alpha^{2}}{25 \lambda} \operatorname{coth} \mu(x-V t)-\frac{3 \alpha^{2}}{25 \lambda} \operatorname{coth}^{2} \mu(x-V t) \text {, } \\
& u_{6}(x, t)=-\frac{3 \alpha^{2}}{25 \lambda}+\frac{6 \alpha^{2}}{25 \lambda} \operatorname{coth} \mu(x-V t)-\frac{3 \alpha^{2}}{25 \lambda} \operatorname{coth}^{2} \mu(x-V t) \text {, } \\
& u_{7}(x, t)=\frac{3 \alpha^{2}}{10 \lambda}-\frac{3 \alpha^{2}}{25 \lambda} \tanh \mu(x-V t)-\frac{3 \alpha^{2}}{100 \lambda} \tanh ^{2} \mu(x-V t) \\
& -\frac{3 \alpha^{2}}{25 \lambda} \operatorname{coth} \mu(x-V t)-\frac{3 \alpha^{2}}{100 \lambda} \operatorname{coth}^{2} \mu(x-V t), \\
& u_{8}(x, t)=-\frac{9 \alpha^{2}}{50 \lambda}-\frac{3 \alpha^{2}}{25 \lambda} \tanh \mu(x-V t)-\frac{3 \alpha^{2}}{100 \lambda} \tanh ^{2} \mu(x-V t) \\
& -\frac{3 \alpha^{2}}{25 \lambda} \operatorname{coth} \mu(x-V t)-\frac{3 \alpha^{2}}{100 \lambda} \operatorname{coth}^{2} \mu(x-V t), \\
& u_{9}(x, t)=\frac{3 \alpha^{2}}{10 \lambda}+\frac{3 \alpha^{2}}{25 \lambda} \tanh \mu(x-V t)-\frac{3 \alpha^{2}}{100 \lambda} \tanh ^{2} \mu(x-V t) \\
& +\frac{3 \alpha^{2}}{25 \lambda} \operatorname{coth} \mu(x-V t)-\frac{3 \alpha^{2}}{100 \lambda} \operatorname{coth}^{2} \mu(x-V t) \\
& u_{10}(x, t)=-\frac{9 \alpha^{2}}{50 \lambda}+\frac{3 \alpha^{2}}{25 \lambda} \tanh \mu(x-V t)-\frac{3 \alpha^{2}}{100 \lambda} \tanh ^{2} \mu(x-V t) \\
& +\frac{3 \alpha^{2}}{25 \lambda} \operatorname{coth} \mu(x-V t)-\frac{3 \alpha^{2}}{100 \lambda} \operatorname{coth}^{2} \mu(x-V t), \\
& u_{11}(x, t)=\frac{9 \alpha^{2}}{25 \lambda}-\frac{6 \alpha^{2}}{25 \lambda} \operatorname{coth} \mu(x-V t)-\frac{3 \alpha^{2}}{25 \lambda} \operatorname{coth}^{2} \mu(x-V t) \text {, } \\
& u_{12}(x, t)=-\frac{3 \alpha^{2}}{25 \lambda}-\frac{6 \alpha^{2}}{25 \lambda} \operatorname{coth} \mu(x-V t)-\frac{3 \alpha^{2}}{25 \lambda} \operatorname{coth}^{2} \mu(x-V t) \text {. }
\end{aligned}
$$

\section{The generalised hyperelastic-rod wave equation}

The generalised hyperelastic-rod wave equation reads as follows:

$$
u_{t}-u_{x x t}+\alpha u_{x}+2 \beta u u_{x}+3 \theta u^{2} u_{x}-\gamma u_{x} u_{x x}-u u_{x x x}=0,
$$

where $\alpha, \beta, \theta$ and $\gamma$ are constant parameters, and we assume that $\theta$ is nonzero. The wave variable $\xi=x-V t$ carries (37) into the ODE

$$
-V U^{\prime}+V U^{\prime \prime \prime}+\alpha U^{\prime}+2 \beta U U^{\prime}+3 \theta U^{2} U^{\prime}-\gamma U^{\prime} U^{\prime \prime}-U U^{\prime \prime \prime}=0 .
$$


Integrating this equation and considering the constant of integration to be zero, we obtain

$$
(-V+\alpha) U+V U^{\prime \prime}+\beta U^{2}+\theta U^{3}-\frac{\gamma-1}{2}\left(U^{\prime}\right)^{2}-U U^{\prime \prime}=0 .
$$

Balancing $U^{3}$ with $U U^{\prime \prime}$ in (39) gives $M=2$. As stated before, the tanh-coth method uses a finite series

$$
U(\mu \xi)=S(Y)=\sum_{k=0}^{2} a_{k} Y^{k}+\sum_{k=1}^{2} b_{k} Y^{-k}
$$

to express the solution $u(x, t)$. Substituting (40) into (39) and collecting the coefficients of $Y$ gives the system of algebraic equations:

$$
\begin{aligned}
& Y^{12}: a_{2}^{3} \theta-4 a_{2}^{2} \mu^{2}-2 a_{2}^{2} \gamma \mu^{2}=0 \\
& Y^{11}: 3 a_{1} a_{2}^{2} \theta-6 a_{1} a_{2} \mu^{2}-2 a_{1} a_{2} \gamma \mu^{2}=0 \text {, } \\
& Y^{10}: 8 a_{2}^{2} \mu^{2}-3 a_{1}^{2} \mu^{2}+2 a_{2}^{2} \beta-a_{1}^{2} \gamma \mu^{2}+8 a_{2}^{2} \gamma \mu^{2}+12 V a_{2} \mu^{2}+6 a_{0} a_{2}^{2} \theta \\
& +6 a_{1}^{2} a_{2} \theta-12 a_{0} a_{2} \mu^{2}=0, \\
& Y^{9}: a_{1}^{3} \theta+2 a_{1} a_{2} \beta+2 V a_{1} \mu^{2}+3 b_{1} a_{2}^{2} \theta-8 b_{1} a_{2} \mu^{2}-2 a_{0} a_{1} \mu^{2}+6 a_{1} a_{2} \mu^{2} \\
& +6 a_{0} a_{1} a_{2} \theta+2 b_{1} a_{2} \gamma \mu^{2}+4 a_{1} a_{2} \gamma \mu^{2}=0, \\
& Y^{8}: 2 a_{1}^{2} \mu^{2}+2 a_{2} \alpha+2 a_{1}^{2} \beta-2 V a_{2}+4 a_{0} a_{2} \beta+2 a_{1}^{2} \gamma \mu^{2}-4 a_{2}^{2} \gamma \mu^{2}-16 V a_{2} \mu^{2} \\
& -6 b_{1} a_{1} \mu^{2}+6 b_{2} a_{2}^{2} \theta-24 b_{2} a_{2} \mu^{2}+6 a_{0} a_{1}^{2} \theta+6 a_{0}^{2} a_{2} \theta \\
& +16 a_{0} a_{2} \mu^{2}+12 b_{1} a_{1} a_{2} \theta+2 b_{1} a_{1} \gamma \mu^{2}+8 b_{2} a_{2} \gamma \mu^{2}=0, \\
& Y^{7}: a_{1} \alpha-V a_{1}+2 b_{1} a_{2} \beta+2 a_{0} a_{1} \beta-2 V a_{1} \mu^{2}+3 b_{1} a_{1}^{2} \theta+14 b_{1} a_{2} \mu^{2}-6 b_{2} a_{1} \mu^{2} \\
& +3 a_{0}^{2} a_{1} \theta+2 a_{0} a_{1} \mu^{2}+6 b_{1} a_{0} a_{2} \theta+6 b_{2} a_{1} a_{2} \theta \\
& -4 b_{1} a_{2} \gamma \mu^{2}+2 b_{2} a_{1} \gamma \mu^{2}-2 a_{1} a_{2} \gamma \mu^{2}=0, \\
& Y^{6}: b_{1}^{2} \mu^{2}+a_{1}^{2} \mu^{2}+2 a_{0} \alpha+2 a_{0}^{3} \theta+2 a_{0}^{2} \beta-2 V a_{0}+4 b_{1} a_{1} \beta+4 b_{2} a_{2} \beta-b_{1}^{2} \gamma \mu^{2} \\
& -a_{1}^{2} \gamma \mu^{2}+4 V b_{2} \mu^{2}+4 V a_{2} \mu^{2}+6 b_{2} a_{1}^{2} \theta+6 b_{1}^{2} a_{2} \theta+12 b_{1} a_{1} \mu^{2}-4 b_{2} a_{0} \mu^{2} \\
& +48 b_{2} a_{2} \mu^{2}-4 a_{0} a_{2} \mu^{2}+12 b_{1} a_{0} a_{1} \theta+12 b_{2} a_{0} a_{2} \theta \\
& -4 b_{1} a_{1} \gamma \mu^{2}-16 b_{2} a_{2} \gamma \mu^{2}=0, \\
& Y^{5}: b_{1} \alpha-V b_{1}+2 b_{1} a_{0} \beta+2 b_{2} a_{1} \beta-2 V b_{1} \mu^{2}+3 b_{1} a_{0}^{2} \theta+3 b_{1}^{2} a_{1} \theta \\
& +2 b_{1} a_{0} \mu^{2}-6 b_{1} a_{2} \mu^{2}+14 b_{2} a_{1} \mu^{2}+6 b_{1} b_{2} a_{2} \theta+6 b_{2} a_{0} a_{1} \theta \\
& -2 b_{1} b_{2} \gamma \mu^{2}+2 b_{1} a_{2} \gamma \mu^{2}-4 b_{2} a_{1} \gamma \mu^{2}=0, \\
& Y^{4}: 2 b_{1}^{2} \mu^{2}+2 b_{2} \alpha+2 b_{1}^{2} \beta-2 V b_{2}+4 b_{2} a_{0} \beta+2 b_{1}^{2} \gamma \mu^{2}-4 b_{2}^{2} \gamma \mu^{2}-16 V b_{2} \mu^{2} \\
& +6 b_{1}^{2} a_{0} \theta+6 b_{2} a_{0}^{2} \theta-6 b_{1} a_{1} \mu^{2}+16 b_{2} a_{0} \mu^{2}+6 b_{2}^{2} a_{2} \theta \\
& -24 b_{2} a_{2} \mu^{2}+12 b_{1} b_{2} a_{1} \theta+2 b_{1} a_{1} \gamma \mu^{2}+8 b_{2} a_{2} \gamma \mu^{2}=0, \\
& Y^{3}: b_{1}^{3} \theta+2 b_{1} b_{2} \beta+2 V b_{1} \mu^{2}+6 b_{1} b_{2} \mu^{2}-2 b_{1} a_{0} \mu^{2}+3 b_{2}^{2} a_{1} \theta-8 b_{2} a_{1} \mu^{2}
\end{aligned}
$$




$$
\begin{gathered}
+6 b_{1} b_{2} a_{0} \theta+4 b_{1} b_{2} \gamma \mu^{2}+2 b_{2} a_{1} \gamma \mu^{2}=0, \\
Y^{2}: 8 b_{2}^{2} \mu^{2}-3 b_{1}^{2} \mu^{2}+2 b_{2}^{2} \beta-b_{1}^{2} \gamma \mu^{2}+8 b_{2}^{2} \gamma \mu^{2}+12 V b_{2} \mu^{2} \\
+6 b_{1}^{2} b_{2} \theta+6 b_{2}^{2} a_{0} \theta-12 b_{2} a_{0} \mu^{2}=0, \\
Y^{1}: 3 b_{1} b_{2}^{2} \theta-6 b_{1} b_{2} \mu^{2}-2 b_{1} b_{2} \gamma \mu^{2}=0, \\
Y^{0}: b_{2}^{3} \theta-4 b_{2}^{2} \mu^{2}-2 b_{2}^{2} \gamma \mu^{2}=0 .
\end{gathered}
$$

The last system gives the three sets of solutions as follows.

The first:

$$
\begin{aligned}
& a_{0}=\left(-8 \gamma \mu^{2}+12 \beta-8 \mu^{2}-40 \beta \mu^{2}+50 \gamma^{3} \mu^{2}+18 \theta \alpha-80 \mu^{2} \beta^{2}-16 \beta \gamma^{2} \mu^{2}\right. \\
& -88 \mu^{2} \gamma \beta^{2}+24 \beta^{2}+32 \gamma \beta^{2}+28 \gamma \beta+26 \gamma^{2} \mu^{2}+14 \gamma^{2} \beta^{2}+33 \theta \alpha \gamma+36 \beta \theta \alpha \\
& +18 \theta \alpha \gamma^{2}-24 \beta^{2} \gamma^{2} \mu^{2}+20 \beta \gamma^{3} \mu^{2}-68 \mu^{2} \gamma \beta+23 \beta \gamma^{2}+24 \mu^{2} \theta \alpha+12 \mu^{2} \theta \alpha \gamma \\
& +30 \beta \theta \alpha \gamma-24 \mu^{2} \theta \alpha \gamma^{2}+6 \beta \theta \alpha \gamma^{2}-12 \gamma^{3} \mu^{2} \theta \alpha+8 \beta \gamma^{4} \mu^{2}+3 \theta \alpha \gamma^{3}+30 \gamma^{4} \mu^{2} \\
& \left.+2 \beta^{2} \gamma^{3}+\beta \gamma^{4}+6 \gamma^{5} \mu^{2}+8 \beta \gamma^{3}\right) \\
& /\left\{2 \theta \left(-12 \gamma^{2}-8 \gamma-2 \beta^{2}-3 \theta \alpha-7 \beta-8 \gamma^{3}-2 \gamma^{4}+24 \beta \gamma^{2} \mu^{2}+4 \beta \gamma^{3} \mu^{2}\right.\right. \\
& +44 \mu^{2} \gamma \beta+24 \beta \mu^{2}-2+34 \gamma^{2} \mu^{2}+14 \gamma^{3} \mu^{2}+2 \gamma^{4} \mu^{2}+34 \gamma \mu^{2}+12 \mu^{2}-4 \gamma \beta^{2} \\
& \left.\left.-2 \gamma^{2} \beta^{2}+3 \theta \alpha \gamma^{2}-16 \gamma \beta-13 \beta \gamma^{2}-4 \beta \gamma^{3}\right)\right\}, \\
& a_{1}=b_{1}=b_{2}=0, \quad a_{2}=\frac{2 \mu^{2}(2+\gamma)}{\theta}, \\
& V=-\left(-8 \gamma \mu^{2}+4 \beta-8 \mu^{2}+8 \beta \mu^{2}+50 \gamma^{3} \mu^{2}+18 \theta \alpha+16 \mu^{2} \beta^{2}+76 \beta \gamma^{2} \mu^{2}\right. \\
& +40 \mu^{2} \gamma \beta^{2}-4 \beta^{2}-8 \beta^{3}-18 \gamma \beta^{2}+26 \gamma^{2} \mu^{2}-20 \gamma^{2} \beta^{2}-12 \gamma \beta^{3}+33 \theta \alpha \gamma \\
& +24 \beta \theta \alpha+18 \theta \alpha \gamma^{2}+32 \beta^{2} \gamma^{2} \mu^{2}+52 \beta \gamma^{3} \mu^{2}+44 \mu^{2} \gamma \beta-13 \beta \gamma^{2}+24 \mu^{2} \theta \alpha \\
& +12 \mu^{2} \theta \alpha \gamma+36 \beta \theta \alpha \gamma-24 \mu^{2} \theta \alpha \gamma^{2}+12 \beta \theta \alpha \gamma^{2}-12 \gamma^{3} \mu^{2} \theta \alpha+12 \beta \mu^{2} \gamma^{4} \\
& \left.+3 \theta \alpha \gamma^{3}+8 \gamma^{3} \mu^{2} \beta^{2}+30 \gamma^{4} \mu^{2}-6 \beta^{2} \mu^{3}-3 \beta \gamma^{4}+6 \gamma^{5} \mu^{2}-12 \beta \gamma^{3}-4 \gamma^{2} \beta^{3}\right) \\
& /\left\{6 \theta \left(-2 \gamma^{3}+2 \gamma^{3} \mu^{2}-6 \gamma^{2}+12 \gamma^{2} \mu^{2}-4 \beta \gamma^{2}+4 \beta \gamma^{2} \mu^{2}-6 \gamma+3 \theta \alpha \gamma-9 \gamma \beta\right.\right. \\
& \left.\left.-2 \gamma \beta^{2}+22 \gamma \mu^{2}+20 \mu^{2} \mu \beta-7 \beta-2 \beta^{2}-3 \theta \alpha+12 \mu^{2}+24 \beta \mu^{2}-2\right)\right\}, \\
& \mu_{1}=\frac{ \pm 1}{4\left(\gamma^{2}+3 \gamma+2\right)}\left(-\left(2 \gamma^{2}+6 \gamma+4\right)\left(-2-\gamma^{2}-4 \beta-3 \gamma-2 \gamma \beta\right.\right. \\
& +\left(4-16 \beta+12 \mu+16 \beta^{2}+13 \gamma^{2}+6 \gamma^{3}+\gamma^{4}+16 \gamma \beta^{2}-32 \gamma \beta+4 \gamma^{2} \beta^{2}\right. \\
& \left.\left.\left.-20 \beta \gamma^{2}-4 \beta \gamma^{3}-48 \theta \alpha-72 \theta \alpha \gamma-24 \theta \alpha \gamma^{2}\right)^{\frac{1}{2}}\right)\right)^{\frac{1}{2}}, \\
& \mu_{2}=\frac{ \pm 1}{4\left(\gamma^{2}+3 \gamma+2\right)}\left(( 2 \gamma ^ { 2 } + 6 \gamma + 4 ) \left(2+\gamma^{2}+4 \beta+3 \gamma+2 \gamma \beta\right.\right. \\
& +\left(4-16 \beta+12 \mu+16 \beta^{2}+13 \gamma^{2}+6 \gamma^{3}+\gamma^{4}+16 \gamma \beta^{2}-32 \gamma \beta+4 \gamma^{2} \beta^{2}\right. \\
& \left.\left.\left.-20 \beta \gamma^{2}-4 \beta \gamma^{3}-48 \theta \alpha-72 \theta \alpha \gamma-24 \theta \alpha \gamma^{2}\right)^{\frac{1}{2}}\right)\right)^{\frac{1}{2}} \text {. }
\end{aligned}
$$


The second:

$$
\begin{aligned}
& a_{0}=\left(-8 \gamma \mu^{2}+12 \beta-8 \mu^{2}-40 \beta \mu^{2}+50 \gamma^{3} \mu^{2}+18 \theta \alpha-80 \mu^{2} \beta^{2}-16 \beta \gamma^{2} \mu^{2}\right. \\
& -88 \mu^{2} \gamma \beta^{2}+24 \beta^{2}+32 \gamma \beta^{2}+28 \gamma \beta+26 \gamma^{2} \mu^{2}+14 \gamma^{2} \beta^{2}+33 \theta \alpha \gamma+36 \beta \theta \alpha \\
& +18 \theta \alpha \gamma^{2}-24 \beta^{2} \gamma^{2} \mu^{2}+20 \beta \gamma^{3} \mu^{2}-68 \mu^{2} \gamma \beta+23 \beta \gamma^{2}+24 \mu^{2} \theta \alpha+12 \mu^{2} \theta \alpha \gamma \\
& +30 \beta \theta \alpha \gamma-24 \mu^{2} \theta \alpha \gamma^{2}+6 \beta \theta \alpha \gamma^{2}-12 \gamma^{3} \mu^{2} \theta \alpha+8 \beta \gamma^{4} \mu^{2}+3 \theta \alpha \gamma^{3}+30 \gamma^{4} \mu^{2} \\
& \left.+2 \beta^{2} \gamma^{3}+\beta \gamma^{4}+6 \gamma^{5} \mu^{2}+8 \beta \gamma^{3}\right) \\
& /\left\{2 \theta \left(-12 \gamma^{2}-8 \gamma-2 \beta^{2}-3 \theta \alpha-7 \beta-8 \gamma^{3}-2 \gamma^{4}+24 \beta \gamma^{2} \mu^{2}+4 \beta \gamma^{3} \mu^{2}\right.\right. \\
& +44 \mu^{2} \gamma \beta+24 \beta \mu^{2}-2+34 \gamma^{2} \mu^{2}+14 \gamma^{3} \mu^{2}+2 \gamma^{4} \mu^{2}+34 \gamma \mu^{2}+12 \mu^{2} \\
& \left.\left.-4 \gamma \beta^{2}-2 \gamma^{2} \beta^{2}+3 \theta \alpha \gamma^{2}-16 \gamma \beta-13 \beta \gamma^{2}-4 \beta \gamma^{3}\right)\right\} \text {, } \\
& a_{1}=a_{2}=b_{1}=0, \quad b_{2}=\frac{2 \mu^{2}(2+\gamma)}{\theta}, \\
& V=-\left(-8 \gamma \mu^{2}+4 \beta-8 \mu^{2}+8 \beta \mu^{2}+50 \gamma^{3} \mu^{2}+18 \theta \alpha+16 \mu^{2} \beta^{2}+76 \beta \gamma^{2} \mu^{2}+40 \mu^{2} \gamma \beta^{2}\right. \\
& -4 \beta^{2}-8 \beta^{3}-18 \gamma \beta^{2}+26 \gamma^{2} \mu^{2}-20 \gamma^{2} \beta^{2}-12 \gamma \beta^{3}+33 \theta \alpha \gamma+24 \beta \theta \alpha+18 \theta \alpha \gamma^{2} \\
& +32 \beta^{2} \gamma^{2} \mu^{2}+52 \beta \gamma^{3} \mu^{2}+44 \mu^{2} \gamma \beta-13 \beta \gamma^{2}+24 \mu^{2} \theta \alpha+12 \mu^{2} \theta \alpha \gamma+36 \beta \theta \alpha \gamma \\
& -24 \mu^{2} \theta \alpha \gamma^{2}+12 \beta \theta \alpha \gamma^{2}-12 \gamma^{3} \mu^{2} \theta \alpha+12 \beta \mu^{2} \gamma^{4}+3 \theta \alpha \gamma^{3}+8 \gamma^{3} \mu^{2} \beta^{2} \\
& \left.+30 \gamma^{4} \mu^{2}-6 \beta^{2} \mu^{3}-3 \beta \gamma^{4}+6 \gamma^{5} \mu^{2}-12 \beta \gamma^{3}-4 \gamma^{2} \beta^{3}\right) \\
& /\left\{6 \theta \left(-2 \gamma^{3}+2 \gamma^{3} \mu^{2}-6 \gamma^{2}+12 \gamma^{2} \mu^{2}-4 \beta \gamma^{2}+4 \beta \gamma^{2} \mu^{2}-6 \gamma+3 \theta \alpha \gamma-9 \gamma \beta\right.\right. \\
& \left.\left.-2 \gamma \beta^{2}+22 \gamma \mu^{2}+20 \mu^{2} \mu \beta-7 \beta-2 \beta^{2}-3 \theta \alpha+12 \mu^{2}+24 \beta \mu^{2}-2\right)\right\}, \\
& \mu_{1}=\frac{ \pm 1}{4\left(\gamma^{2}+3 \gamma+2\right)}\left(-\left(2 \gamma^{2}+6 \gamma+4\right)\left(-2-\gamma^{2}-4 \beta-3 \gamma-2 \gamma \beta\right.\right. \\
& +\left(4-16 \beta+12 \mu+16 \beta^{2}+13 \gamma^{2}+6 \gamma^{3}+\gamma^{4}+16 \gamma \beta^{2}-32 \gamma \beta+4 \gamma^{2} \beta^{2}\right. \\
& \left.\left.\left.-20 \beta \gamma^{2}-4 \beta \gamma^{3}-48 \theta \alpha-72 \theta \alpha \gamma-24 \theta \alpha \gamma^{2}\right)^{\frac{1}{2}}\right)\right)^{\frac{1}{2}}, \\
& \mu_{2}=\frac{ \pm 1}{4\left(\gamma^{2}+3 \gamma+2\right)}\left(( 2 \gamma ^ { 2 } + 6 \gamma + 4 ) \left(2+\gamma^{2}+4 \beta+3 \gamma+2 \gamma \beta\right.\right. \\
& +\left(4-16 \beta+12 \mu+16 \beta^{2}+13 \gamma^{2}+6 \gamma^{3}+\gamma^{4}+16 \gamma \beta^{2}-32 \gamma \beta+4 \gamma^{2} \beta^{2}\right. \\
& \left.\left.\left.-20 \beta \gamma^{2}-4 \beta \gamma^{3}-48 \theta \alpha-72 \theta \alpha \gamma-24 \theta \alpha \gamma^{2}\right)^{\frac{1}{2}}\right)\right)^{\frac{1}{2}} \text {. }
\end{aligned}
$$

The third:

$$
\begin{aligned}
a_{0}= & \left(-8 \gamma \mu^{2}+3 \beta-8 \mu^{2}-40 \beta \mu^{2}+50 \gamma^{3} \mu^{2}+\frac{9}{2} \theta \alpha-80 \mu^{2} \beta^{2}-16 \beta \gamma^{2} \mu^{2}\right. \\
& -88 \mu^{2} \gamma \beta^{2}+6 \beta^{2}+8 \gamma \beta^{2}+7 \gamma \beta+26 \gamma^{2} \mu^{2}+\frac{7}{2} \gamma^{2} \beta^{2}+\frac{33}{4} \theta \alpha \gamma+9 \beta \theta \alpha \\
& -24 \beta^{2} \gamma^{2} \mu^{2}+20 \beta \gamma^{3} \mu^{2}-68 \mu^{2} \gamma \beta+\frac{23}{4} \beta \gamma^{2}+24 \mu^{2} \theta \alpha+12 \mu^{2} \theta \alpha \gamma+\frac{15}{2} \beta \theta \alpha \gamma \\
& -24 \mu^{2} \theta \alpha \gamma^{2}+\frac{3}{2} \beta \theta \alpha \gamma^{2}-12 \gamma^{3} \mu^{2} \theta \alpha+8 \beta \gamma^{4} \mu^{2}+\frac{3}{4} \theta \alpha \gamma^{3}+30 \gamma^{4} \mu^{2}+\frac{1}{2} \beta^{2} \gamma^{3}
\end{aligned}
$$




$$
\begin{aligned}
& \left.+\frac{1}{4} \beta \gamma^{4}+6 \gamma^{5} \mu^{2}+2 \beta \gamma^{3}\right) \\
& /\left\{\theta \left(-2+16 \beta \gamma^{3} \mu^{2}+176 \mu^{2} \gamma \beta-12 \gamma^{2}-8 \gamma-3 \theta \alpha-2 \beta^{2}-8 \gamma^{3}-2 \gamma^{4}-7 \beta\right.\right. \\
& +48 \mu^{2}+96 \beta \gamma^{2} \mu^{2}+3 \theta \alpha \gamma^{2}-16 \gamma \beta-13 \beta \gamma^{2}-4 \beta \gamma^{3}-4 \gamma \beta^{2}-2 \gamma^{2} \beta^{2} \\
& \left.\left.+136 \gamma^{2} \mu^{2}+56 \gamma^{3} \mu^{2}+8 \gamma^{4} \mu^{2}+136 \gamma \mu^{2}+96 \beta \mu^{2}\right)\right\}, \\
& a_{1}=b_{1}=0, \quad a_{2}=b_{2}=\frac{2 \mu^{2}(2+\gamma)}{\theta}, \\
& V=-\left(-32 \gamma \mu^{2}+4 \beta-32 \mu^{2}+32 \beta \mu^{2}+200 \gamma^{3} \mu^{2}+18 \theta \alpha+64 \mu^{2} \beta^{2}+304 \beta \gamma^{2} \mu^{2}\right. \\
& +160 \mu^{2} \gamma \beta^{2}-4 \beta^{2}-8 \beta^{3}-18 \gamma \beta^{2}+104 \gamma^{2} \mu^{2}-20 \gamma^{2} \beta^{2}-12 \gamma \beta^{3}+33 \theta \alpha \gamma \\
& +24 \beta \theta \alpha+18 \theta \alpha \gamma^{2}+128 \beta^{2} \gamma^{2} \mu^{2}+208 \beta \gamma^{3} \mu^{2}+176 \mu^{2} \gamma \beta-13 \beta \gamma^{2}+96 \mu^{2} \theta \alpha \\
& +48 \mu^{2} \theta \alpha \gamma+36 \beta \theta \alpha \gamma-96 \mu^{2} \theta \alpha \gamma^{2}+12 \beta \theta \alpha \gamma^{2}-48 \gamma^{3} \mu^{2} \theta \alpha+48 \beta \mu^{2} \gamma^{4} \\
& \left.+3 \theta \alpha \gamma^{3}+32 \gamma^{3} \mu^{2} \beta^{2}+120 \gamma^{4} \mu^{2}-6 \beta^{2} \mu^{3}-3 \beta \gamma^{4}+24 \gamma^{5} \mu^{2}-12 \beta \gamma^{3}-4 \gamma^{2} \beta^{3}\right) \\
& /\left\{\left(6 \theta 8 \gamma^{3} \mu^{2}-2 \gamma^{3}-6 \gamma^{2}+48 \gamma^{2} \mu^{2}-4 \beta \gamma^{2}+16 \beta \gamma^{2} \mu^{2}-6 \gamma+3 \theta \alpha \gamma-9 \gamma \beta\right.\right. \\
& \left.\left.-2 \gamma \beta^{2}+88 \gamma \mu^{2}+80 \mu^{2} \mu \beta-7 \beta-2 \beta^{2}-3 \theta \alpha+48 \mu^{2}+96 \beta \mu^{2}-2\right)\right\}, \\
& \mu_{1}=\frac{ \pm 1}{8\left(\gamma^{2}+3 \gamma+2\right)}\left(-\left(2 \gamma^{2}+6 \gamma+4\right)\left(-2-\gamma^{2}-4 \beta-3 \gamma-2 \gamma \beta\right.\right. \\
& +\left(4-16 \beta+12 \mu+16 \beta^{2}+13 \gamma^{2}+6 \gamma^{3}+\gamma^{4}+16 \gamma \beta^{2}-32 \gamma \beta+4 \gamma^{2} \beta^{2}\right. \\
& \left.\left.\left.-20 \beta \gamma^{2}-4 \beta \gamma^{3}-48 \theta \alpha-72 \theta \alpha \gamma-24 \theta \alpha \gamma^{2}\right)^{\frac{1}{2}}\right)\right)^{\frac{1}{2}}, \\
& \mu_{2}=\frac{ \pm 1}{8\left(\gamma^{2}+3 \gamma+2\right)}\left(( 2 \gamma ^ { 2 } + 6 \gamma + 4 ) \left(2+\gamma^{2}+4 \beta+3 \gamma+2 \gamma \beta\right.\right. \\
& +\left(4-16 \beta+12 \mu+16 \beta^{2}+13 \gamma^{2}+6 \gamma^{3}+\gamma^{4}+16 \gamma \beta^{2}-32 \gamma \beta+4 \gamma^{2} \beta^{2}\right. \\
& \left.\left.\left.-20 \beta \gamma^{2}-4 \beta \gamma^{3}-48 \theta \alpha-72 \theta \alpha \gamma-24 \theta \alpha \gamma^{2}\right)^{\frac{1}{2}}\right)\right)^{\frac{1}{2}} \text {. }
\end{aligned}
$$

These in turn give the following three solutions:

$$
\begin{aligned}
& u_{1}(x, t)=a_{0}+\frac{2 \mu^{2}(2+\gamma)}{\theta} \tanh ^{2} \mu(x-V t), \\
& u_{2}(x, t)=a_{0}+\frac{2 \mu^{2}(2+\gamma)}{\theta} \operatorname{coth}^{2} \mu(x-V t), \\
& u_{3}(x, t)=a_{0}+\frac{2 \mu^{2}(2+\gamma)}{\theta} \tanh ^{2} \mu(x-V t)+\frac{2 \mu^{2}(2+\gamma)}{\theta} \operatorname{coth}^{2} \mu(x-V t) .
\end{aligned}
$$

\section{Conclusion}

In this paper, we focused on travelling wave solutions of the general form of BenjaminBona-Mahony-Peregrine-Burgers equation, the general form of the Oskolkov-BenjaminBona-Mahony-Burgers equation, the one-dimensional Oskolkov equation and the generalised hyperelastic-rod wave equation. We derived various exact travelling wave solutions of these physical structures by using the tanh-coth method. Throughout the work, Maple was used to deal with the tedious algebraic operations. 


\section{Competing interests}

The authors declare that they have no competing interests.

\section{Authors' contributions}

All authors read and approved the final manuscript.

\section{Acknowledgements}

Dedicated to Professor Hari M Srivastava.

Received: 3 December 2012 Accepted: 7 May 2013 Published: 23 May 2013

\section{References}

1. Quarteroni, A: Fourier spectral methods for pseudo-parabolic equations. SIAM J. Numer. Anal. 24(2), $323-335$ (1987)

2. Korpusov, MO, Sveshnikov, AG: Blow-up of solutions of strongly nonlinear equations of pseudoparabolic type. J. Math. Sci. 148(1), 1-142 (2008)

3. Dubey, SA: Numerical solution for nonlocal Sobolev-type differential equations. Electron. J. Differ. Equ. Conf. 19, 75-83 (2010)

4. Kaikina, El, Naumkin, PI, Shishmarev, IA: The Cauchy problem for an equation of Sobolev type with power non-linearity. Izv. Math. 69(1), 59-111 (2005)

5. Karch, G: Asymptotic behaviour of solutions to some pseudoparabolic equations. Math. Methods Appl. Sci. 20, 271-289 (1997)

6. Peregrine, DH: Calculations of the development of an undular bore. J. Fluid Mech. 25, 321-330 (1996)

7. Benjamin, TB, Bona, JL, Mahony, JJ: Model equations for long waves in nonlinear dispersive systems. Philos. Trans. R. Soc. Lond. Ser. A 272, 47-78 (1972)

8. Camassa, R, Holm, DD: An integrable shallow water equation with peaked solitons. Phys. Rev. Lett. 71(11), 1661-1664 (1993)

9. Meiss, J, Horton, W: Fluctuation spectra of a drift wave soliton gas. Phys. Fluids 25, 1838 (1982)

10. Yam, C: Regularized long wave equation and inverse scattering transform. J. Math. Phys. 34, 2618 (1993)

11. Musette, M, Lambert, F, Decuyper, J: Soliton and antisoliton resonant interactions. J. Phys. A 20, 6223 (1987)

12. Rollins, D: Painlevé analysis and Lie group symmetries of the regularized long-wave equation. J. Math. Phys. 32,3331 (1991)

13. Bona, J, Pritchard, W, Scott, L: Solitary-wave interaction. Phys. Fluids 23, 438 (1980)

14. Kodama, Y: On solitary-wave interaction. Phys. Lett. A 123, 276 (1987)

15. Hereman, W, Banerjee, P, Korpel, A, Assanto, G, Van Immerzeele, A, Meerpoel, A: J. Phys. A 19, 607 (1986)

16. Oskolkov, AP: Nonlocal problems for one class of nonlinear operator equations that arise in the theory of Sobolev-type equations. Zap. Nauč. Semin. POMI 198, 31-48 (1991)

17. Oskolkov, AP: On stability theory for solutions of semilinear dissipative equations of the Sobolev type. Zap. Nauč. Semin. POMI 200, 139-148 (1992)

18. Camassa, R, Holm, DD, Hyman, J: A new integrable shallow water equation. Adv. Appl. Mech. 31, 1-33 (1994)

19. Parkes, EJ, Vakhnenko, VO: Explicit solutions of the Camassa-Holm equation. Chaos Solitons Fractals 26(5), 1309-1316 (2005)

20. Wazwaz, AM: New solitary wave solutions to the modified forms of Degasperis-Procesi and Camassa-Holm equations. Appl. Math. Comput. 186, 130-141 (2007)

21. Johnson, RS: Camassa-Holm, Korteweg-de Vries and related models for water waves. J. Fluid Mech. 455, 63 (2002)

22. Johnson, RS: The classical problem of water waves: a reservoir of integrable and nearly-integrable equations. J. Nonlinear Math. Phys. 10(Suppl. 1) 72 (2003)

23. Amfilokhiev, VB, Voitkunskii, YI, Mazaeva, NP, Khodorkovskii, YS: Flows of polymer solutions in the case of convective accelerations. Tr. Leningr. Korablestroit. Inst. 96, 3-9 (1975)

24. Sviridyuk, GA, Yakupov, MM: The phase space of Cauchy-Dirichlet problem for a nonclassical equation. Differ. Uravn. (Minsk) 39(11), 1556-1561 (2003)

25. Coclite, GM, Holden, H, Karlsen, $\mathrm{KH}$ : Global weak solutions to a generalized hyperelastic-rod wave equation. SIAM J. Math. Anal. 37(4), 1044-1069 (2005)

26. Dulin, HR, Gottwald, GA, Holm, DD: On asymptotically equivalent shallow water wave equations. Physica D 190, 1 (2004)

27. Whitham, GB: Variational methods and applications to water wave. Proc. R. Soc. Lond. A 299, 625 (1967)

28. Fornberg, B, Whitham, GB: A numerical and theoretical study of certain nonlinear wave phenomena. Philos. Trans. R. Soc. Lond. A 289, 373-404 (1978)

29. Gözükızıl, ÖF, Akçağıl, Ş: Exact solutions of Benjamin-Bona-Mahony-Burgers-type nonlinear pseudo-parabolic equations. Bound. Value Probl. 2012, 144 (2012)

30. Gözükızıl, ÖF, Akçağıl, Ş: Travelling wave solutions to the Benney-Luke and the higher-order improved Boussinesq equations of Sobolev type. Abstr. Appl. Anal. 2012, Article ID 890574 (2012)

31. Wazwaz, AM, Helal, MA: Nonlinear variants of the BBM equation with compact and noncompact physical structures. Chaos Solitons Fractals 26(3), 767-776 (2005)

32. Wazwaz, AM: Two reliable methods for solving variants of the KdV equation with compact and non compact structures. Chaos Solitons Fractals 28(2), 454-462 (2006) 